\title{
Impact of the introduction of a novel automated embedding system on quality in a university hospital histopathology department
}

Sine M. Phelan

Correspondence: sine.phelan@hse.ie

CrossMark

$\leftarrow$ Click for updates

Division of Anatomic pathology, University Hospital Galway and National University of Ireland, Galway, Ireland.

\begin{abstract}
Background: Automation of tasks in the laboratory can improve efficiency and quality of service. In light of increased pressure on resources an automated Embedder (Autotec ${ }^{\circledR}$ ) was purchased, with the aim of reducing staff required for embedding and improving Turn Around Times (TAT).

Design: Following a verification process, all resections, LLETZ specimens, curettings and skin excisions $>2 \mathrm{~cm}$ were processed using the Autotec ${ }^{\oplus}$ such that it was used for at least $50 \%$ of blocks daily. The number of scientific staff required for embedding each day was compared before introduction of the Autotec ${ }^{\circledR}$ and after achieving 50\% throughput. Block numbers were also compared before and after its introduction. Pathologists were surveyed regarding the impact on workflow in the cut-up room and on the quality of sections.

Results: TAT improved by 4 days for cancer resections and endoscopic biopsies, and by 5 days for surgical specimens NOS. Time rostered for embedding was reduced from 5.5 hours to 3 hours/day. Block numbers did not increase significantly $(<1 \%)$ following introduction of the Autotec ${ }^{\oplus}$. Of nine pathologists who were surveyed, 4 believed that the Autotec ${ }^{\circledast}$ had increased the time spent in cut up, ranging from a slight increase up to $25 \%, 2$ reported that the time spent had not increased and the remaining 3 did not specify. Five of 9 reported a minor negative impact on the quality of sections, without impact on histological evaluation e.g. edge artefact.

Conclusions: Introduction of the Autotec ${ }^{\oplus}$ lead to dramatic improvements in TAT, without increased scientific staff. Its use increased the amount of time spent by pathologists in the cut-up room, possibly negatively impacting the training of resident staff.

Keywords: Automation, quality assurance, histology, endoscopic biopsies
\end{abstract}

\section{Introduction}

In general, automation of laboratory processes increases efficiency and reduces both error rates and costs [1,2]. Advances in information technology, quality control and quality assurance methods have also made a valuable contribution in reducing diagnostic errors [3]. Histology remains a highly manual discipline in comparison to other areas of Laboratory Medicine, which is dependent on the availability of well-trained and experienced technical staff. Recently, a fully automated system for tissue embedding has been developed (Tissue-Tek ${ }^{\circledast}$ AutoTEC ${ }^{\circledast}$ ). This system allows tissue pieces to be orientated within specially designed cassette inserts (Figure 1). These inserts are made form a sectionable material (Paraform ${ }^{\circledR}$ ) and are available in 6 types, which can accommodate a variety of tissue sizes from large sections to small biopsies and skin pieces (Figure 2). The tissue remains stable within the cassette insert throughout processing and paraffin wax is automatically inserted around the tissue. Section cutting commences directly after processing, eliminating the need for manual embedding. The block is moved therefore directly from the processor to the microtomy station. The cassette base, composed of the insert, is sectioned through at the microtome, until the tissue becomes visible and the tissue is then sectioned in the conventional manner.
Our laboratory is based in regional cancer centre, with a workload of approximately 32,000 surgical specimens per year. In light of increased pressure on resources, primarily staffing, within our department, which led to an unacceptable delay in the throughput of specimens, a decision was made to purchase this automated system, with the aim of improving Turn-Around Times (TAT) without increasing staff numbers or impacting section quality. The aim of this study is to assess the benefits of automation of embedding in histology and to identify any possible negative impact on Pathologists' workflow at the cut-up bench and on section quality.

\section{Materials and methods}

Verification process

Following purchase of the automated system a verification period commenced. Specimens considered to be low risk for embedding difficulties were selected. These included specimens for which additional tissue would be available in the event of poor quality of initial sections or specimens that do not routinely require orientation. Initially bowel resections and hysterectomies for benign disease and Trans Urethral Resection of Prostate (TURP) were utilised. The resultant sections were reviewed by a Pathologist with a special interest in the specified 


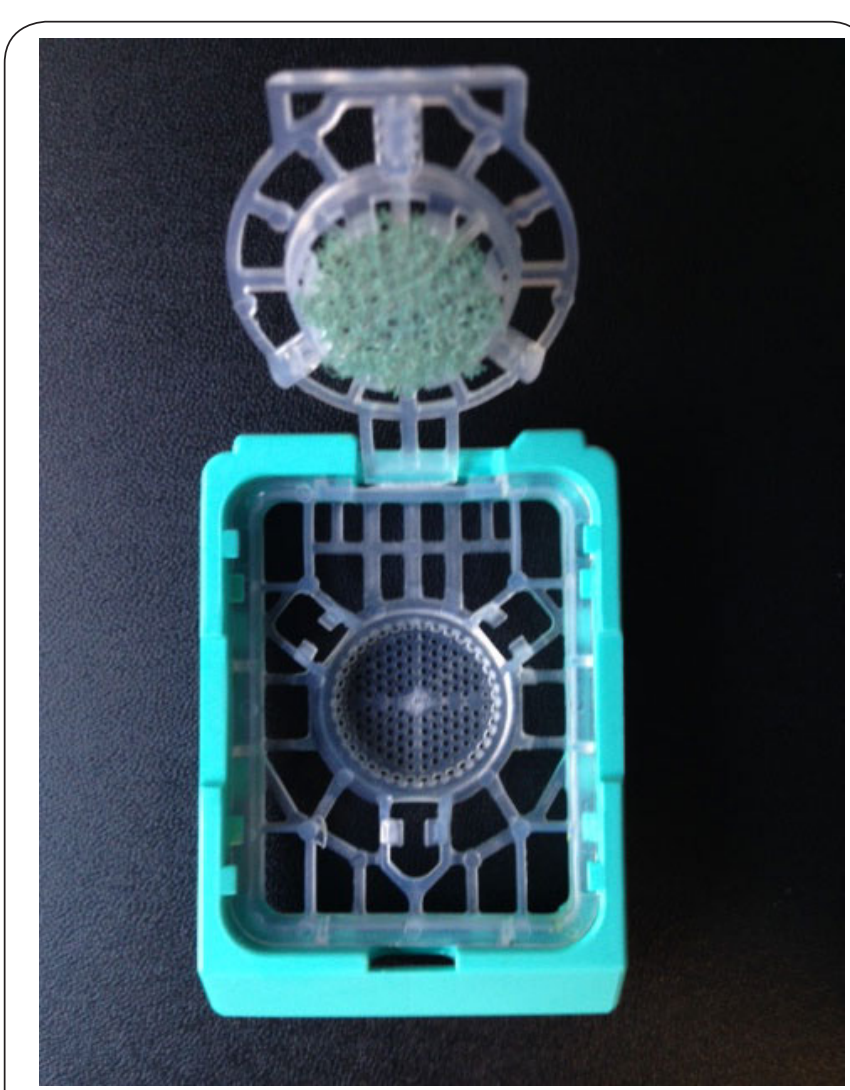

Figure 1. Specially designed cassette inserts made from sectionable material are used.

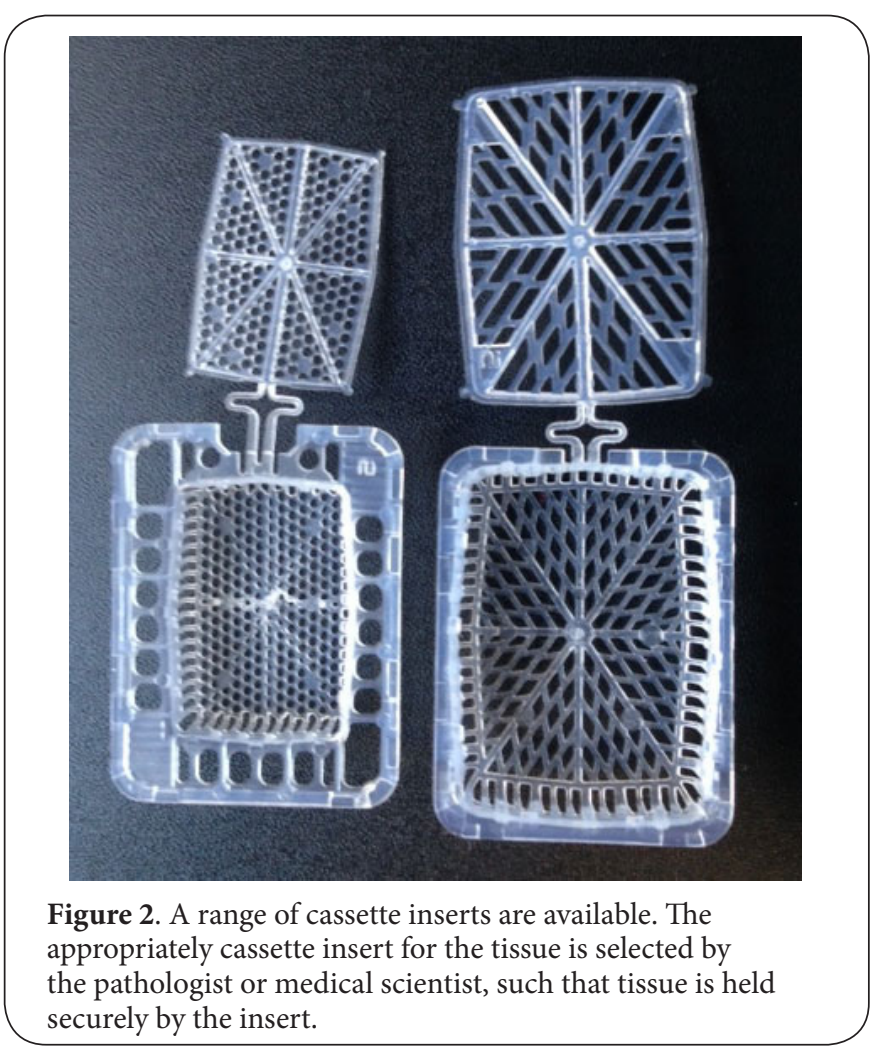

area and any problems reported to the Chief Medical Scientist. As confidence increased in the section quality obtained from the automatic embedder, the volume and range of specimens was increased to include all LLETZ specimens, curettings, the majority of large resections and skin excisions measuring $>2 \mathrm{~cm}$, such that $>50 \%$ of all blocks were processed on the automated embedder.

Initially one Pathologist, assisted by a Medical Laboratory Aid, was trained in the selection and use of cassette inserts. Onsite training was provided by the company (Sakura) for one week. Cascade training then proceeded over a period of 6 weeks until all Pathologists and Medical Science staff involved in grossing had gained some experience of the system.

TATs and block numbers for a 3 month period, were compared before introduction of the Autotec ${ }^{\circledast}$ and after achieving $50 \%$ throughput. TAT was calculated from the Laboratory information System and was based on the day on which $>80 \%$ of cases were authorised.

Pathologists were anonymously surveyed regarding the impact of the automated system on workflow in the cut-up room and on the quality of sections. They were asked whether use of the automated system had increased the time spent in cut-up and if so how much additional time was spent. They were also asked whether the system had impacted on section quality.

\section{Results}

\section{Review staff and block numbers}

Time rostered for embedding was reduced from 5.5 hours to 3 hours/day, freeing more Medical Scientists for other duties, primarily microtomy.

Specimen numbers remained broadly similar. Block numbers increased overall by $<1 \%$ and ranged from 1,500 to 2,600 per week during both time periods.

There was no increase in the number of Medical Scientists or Pathologists.

Impact on pathologists-grossing and section quality Nine of 11 pathologists responded to the survey. Four believed that the automated system had increased the time spent in cut up, ranging from a slight increase up to $25 \%$. Two reported that the time spent had not increased and the remaining 3 did not specify. Five of 9 reported a minor negative impact on the quality of sections, without impact on histological evaluation. The most frequently cited issue was "edge artefact", in which the edge of the section appears corrugated due to squeezing of the tissue within the cassette. This artefact occurs when the insert used is too small for the tissue. One pathologist commented that because a smaller volume of material is present in each block, the number of blocks submitted was increased.

\section{Discussion}

In general, automation of processes leads to increased efficiency 
and reduced risk of error, provided that quality can be maintained [2]. Complexity of processes in the laboratory is regarded as increasing risk of error; with every step in a process the risk of error increases and it has been estimated that if there is a $1 \%$ error rate at each step, with 25 steps the risk of error goes up to $22 \%$ [4]. Histopathology has numerous steps in receiving, processing, and reporting a specimen. It remains a highly complex and manual discipline, requiring significant operator skill and experience. Correct orientation of tissue samples is often a prerequisite for accurate diagnosis [5] and the concept of automation of the embedding of tissue blocks is met with some trepidation by many Pathologists for this reason. Errors can be detected in each phase within surgical pathology: accessioning, gross dissecting, processing, embedding , tissue cutting and slide mounting, coloring, labeling and releasing [6] and automation of any of these processes has the potential to reduce error.

Introduction of automation of embedding constitutes a significant change in work practise within the grossing room. The cassettes used are quite different from the conventional kind, in that they are relatively pliable and require precision when closing. If the lid is pushed down too forcefully, the tissue is compressed into the cassette too deeply and cannot be subsequently sectioned, leading to a requirement for re-embedding. If the lid is not closed tightly enough, it risks opening whilst in the machine, with loss of tissue. Clearly, this has the potential to significantly delay progress in the cut-up room and for this reason, the change was resisted by many pathologists-in-training within our department, particularly those who were more senior and felt their efficiency in the cut-up room was negatively impacted. Our approach to this was to ensure a well trained Laboratory Aid assisted the Pathologist at all times within the cut-up room and took responsibility for the closing of cassettes. As staff became more experienced with the system and accustomed to the new cassettes, this became less of an issue. However, as borne out by the survey undertaken of Pathologists' views of this system, use of the automated system appeared to increase the time spent in the cut-up room. This is particularly an issue for pathologists in-training, who will consequently have less time available for microscopy and whose overall progress in training may suffer.

In relation to large resections, there was some concern that use of the new system would increase block numbers. The cassette inserts are smaller than a conventional cassette. It should be noted however that the inserts were specifically designed to mirror the optimum block size recommended for a conventional cassette. Whereas a conventional cassette ideally should not be entirely filled by tissue, the tissue should be flush with the edges of the insert in an automated cassette. This removes the option of overfilling a cassette from the Pathologist, which could be regarded as an advantage, reducing the likelihood of blocks requiring re-processing. Although some Pathologists perceived an increase in block numbers resulting from the new system, this was not borne out by the evidence. Block numbers did not increase significantly $(<1 \%)$. Megablocks are also available in our laboratory. The use of megablocks where a large section is required, in combination with the automated system provides the pathologist with sufficient flexibility for most cases.

The specially designed cassette inserts are made from an inert sectionable material. Therefore, rather than lids being removed from cassettes, they are sectioned through at the microtome. This may increase the time taken at microtomy for each tissue block. Medical Scientists in our laboratory were unable to specify how much extra time is taken.

It could be argued that automation of embedding in our laboratory has occurred at the expense of time in the grossing room and time at microtomy. Despite these issues, Turn-Around Times have markedly improved following introduction of this system. Although increased time is devoted to two other steps in the chain, the complete removal of this one step, with associated ancillary delays, for approximately $50 \%$ of cases, has had a positive effect. This is in line with Lean principals [7]. It should be noted that this system was introduced into our laboratory during a time of significant pressure on staffing resources which had led to a backlog of uncut blocks, which accounts for the dramatic improvement in TAT.

Introduction of the Autotec ${ }^{\circledast}$ lead to dramatic improvements in TAT, without increased scientific staff. Its use increased the amount of time spent by pathologists in the cut-up room, possibly negatively impacting the training of junior staff. This has been a valuable addition to our laboratory but represented a major change in work practices and therefore required a cautious and methodical introduction. There remains a requirement for manual embedding of small specimens for which orientation and preservation of tissue is paramount.

\section{Competing interests}

The author declares that she has no competing interests.

\section{Acknowledgement}

The author would like to acknowledge Ms. Terri Muldoon, Chief Medical Scientist, UCHG and Ms. Frances Devlin, Senior Medical Scientist, UCHG, for assistance in compiling data.

\section{Publication history}

EIC: Giuseppe Musumeci, University of Catania, Italy.

Received: 25-Mar-2014 Final Revised: 09-Apr-2014

Accepted: 23-Apr-2014 Published: 22-May-2014

\section{References}

1. Novis DA. Detecting and preventing the occurrence of errors in the practices of laboratory medicine and anatomic pathology: 15 years' experience with the College of American Pathologists' Q-PROBES and Q-TRACKS programs. Clin Lab Med. 2004; 24:965-78. I Article I PubMed

2. Welch DL. Human error and human factors engineering in health care. Biomed Instrum Technol. 1997; 31:627-31. I PubMed

3. Lippi G, Becan-McBride K, Behulova D, Bowen RA, Church S, Delanghe J, Grankvist K, Kitchen S, Nybo M, Nauck M, Nikolac N, Palicka V, Plebani $M$, Sandberg $S$ and Simundic AM. Preanalytical quality improvement: 
Sine M. Phelan, Journal of Histology \& Histopathology 2014,

http://www.hoajonline.com/journals/pdf/2055-091X-1-3.pdf

in quality we trust. Clin Chem Lab Med. 2013; 51:229-41. | Article |

PubMed

4. Nakhleh RE. Patient safety and error reduction in surgical pathology. Arch Pathol Lab Med. 2008; 132:181-5. | Article | PubMed

5. Trongwongsa T, Tanboon J, Nimmannit A and Pongpaibul A. The specimen handling of GI mucosal biopsy: a simple and effective quality improvement initiative. J Med Assoc Thai. 2013; 96:1374-9. | PubMed

6. Morelli P, Porazzi E, Ruspini M, Restelli U and Banfi G. Analysis of errors in histology by root cause analysis: a pilot study. J Prev Med Hyg. 2013; 54:90-6. | Pdf | PubMed

7. Serrano L, Hegge P, Sato B, Richmond B and Stahnke L. Using LEAN principles to improve quality, patient safety, and workflow in histology and anatomic pathology. Adv Anat Pathol. 2010; 17:215-21. | Article | PubMed

Citation:
Phelan SM. Impact of the introduction of a novel
automated embedding system on quality in a
university hospital histopathology department. J
Histol Histopathol. 2014; 1:3.
http://dx.doi.org/10.7243/2055-091X-1-3

